\title{
First demonstration and performance of an injection locked continuous wave magnetron to phase control a superconducting cavity
}

\author{
A. C. Dexter, G. Burt, R. G. Carter, and I. Tahir \\ Engineering Department, Lancaster University, Lancaster, LA1 4YR, United Kingdom \\ and Cockcroft Institute, Daresbury, Warrington, United Kingdom \\ H. Wang, K. Davis, and R. Rimmer \\ Thomas Jefferson Lab, Newport News, Virginia 23606, USA \\ (Received 18 October 2010; published 17 March 2011)
}

\begin{abstract}
The applications of magnetrons to high power proton and cw electron linacs are discussed. An experiment is described where a $2.45 \mathrm{GHz}$ magnetron has been used to drive a single cell superconducting cavity. With the magnetron injection locked, a modest phase control accuracy of $0.95^{\circ} \mathrm{rms}$ has been demonstrated. Factors limiting performance have been identified.
\end{abstract}

DOI: $10.1103 /$ PhysRevSTAB.14.032001

PACS numbers: 84.40.Fe, 29.20.-c

\section{INTRODUCTION}

This paper describes the potential application of magnetron rf power sources to proton or electron linacs and describes a proof of principle experiment. Magnetrons are widely regarded as noisy oscillators and, hence, would not normally be considered as suitable for driving accelerator cavities whose phase and amplitude must be accurately controlled with respect to adjacent cavities when powered from separate sources. For the experimental work presented here, the magnetron is operated as an amplifier in a feedback loop and the cathode heater power is adjusted to allow the magnetron to operate in a relatively low noise state. In this mode of operation, the performance of the magnetron was sufficient for the phase of a high $Q$ superconducting cavity to be controlled to better than $1^{\circ}$ in the presence of cavity microphonics. This is the first time that phase control of a superconducting cavity has been attempted and indeed achieved using a magnetron as the amplifier.

New, high power, long pulse, superconducting proton linacs are being proposed as drivers for key science experiments including neutrino factories, EURISOL, the European Spallation Source, and the LHC luminosity upgrade (SPL) [1,2]. Continuous wave (cw) superconducting electron linacs are under development, for example CHESS at CESR [3], or being upgraded, for example CEBAF to $12 \mathrm{GeV}$ [4]. Key issues for such constructions, their operation, and lifetime maintenance are cost and efficient $\mathrm{rf}$ generation. Appropriate to the rf power and frequency requirement, klystron amplifiers or inductive output tube amplifiers (IOT) would normally be utilized.

Published by American Physical Society under the terms of the Creative Commons Attribution 3.0 License. Further distribution of this work must maintain attribution to the author(s) and the published article's title, journal citation, and DOI.
Klystrons offer much higher output powers and gain than IOTs. Conventional klystron sources typically offer electronic efficiencies up to 65\% [5] with a tube cost of about $\$ 200$ per $\mathrm{kW}$ based on a $2 \mathrm{MW}, 10 \%$ duty cycle tube at $700 \mathrm{MHz}$. IOTs in use for light source facilities typically operate with efficiencies of about 65\% [6] and have a cost per $\mathrm{kW}$ which is greater than high power klystrons. In principle, this efficiency can be increased using depressed collectors but this adds considerable cost to the power supply. In contrast, magnetrons offer efficiencies up to $90 \%$ [7] and a substantially lower tube cost which is about $\$ 120$ per kW for $915 \mathrm{MHz}$ cw tubes. The magnetron cost saving comes predominantly from its much smaller size. Other savings come from its high efficiency reducing the cooling requirement and the low anode voltage reducing $\mathrm{x}$-ray shielding requirements. Anticipating minimal maintenance, the magnetron could typically fit alongside superconducting cavities in the same tunnel and where this is acceptable with respect to any such maintenance, then huge civil engineering costs might also be saved.

The proton linacs of interest have typical rf peak power requirements in the GW range and, hence, will always need multiple sources for many superconducting cavities. The electron linacs of interest $[3,4]$ have typical $\mathrm{cw}$ power requirements in the range of tens of $\mathrm{kW}$ or less; however, one power source per superconducting cavity is preferred. Electricity cost savings are huge for the new generation of proton drivers and significant for the $\mathrm{cw}$ electron linacs.

The maximum long pulse output power for a magnetron that is reliably achievable is typically an order of magnitude less than that for a klystron but is significantly larger than that for an IOT. Magnetron design for output powers sufficient to drive individual multicell cavities for a range of current projects such as SPL or ERLs is well within the state of the art.

Correct operation will always require precise amplitude and phase control of successive cavities; for the SPL 
simulations [8] amplitude errors of $\pm 1 \%$ and phase errors of \pm 1 degree on the $\mathrm{rf}$ fields in the superconducting cavities were assumed and consequential emittance growth and energy spread was acceptable. Cavity phase and amplitude is perturbed by beamloading and microphonics. Consequently, one must be able to vary the amplitude and phase from each rf power source for dynamic compensation so that each cavity closely maintains the optimum phase and amplitude for design gradient and beam stability.

Early magnetrons were investigated as both oscillators [9] and amplifiers [10]. The theory of phase locked oscillators developed by Adler [11] was immediately applicable to the magnetron and, hence, from this time it was expected that the phase of a magnetron could be locked and, hence, controlled by an injection signal. In the past, short pulse, high power, injection locked, relativistic magnetrons have been considered by Varian as potential rf sources for normal conducting accelerators [12-17]. Two obstacles encountered were the amount of locking power required and the magnetron's narrow instantaneous bandwidth. Before the consideration of $\mathrm{rf}$ sources for accelerators, phase locked magnetrons had been investigated for radar applications [18].

\section{MAGNETRON OPERATION AND LOCKING}

A cavity magnetron has a central cathode in the form of a cylindrical rod or helical filament which emits electrons. These electrons circulate around the cathode as a consequence of an axially applied magnetic field. The magnetron cavity has a slow wave anode structure that surrounds the cathode. This structure allows circulating rf waves to interact with circulating electrons. The rf energy builds up in the structure and is extracted to the external circuit by couplers in one or more of the cavity cells. The frequency of the magnetron's output is constrained by the bandwidth of the resonant mode but also depends on the cathode voltage relative to the anode, the applied magnetic field, the charge density in the rotating electron cloud, and the level of excitation of the slow rf wave. The relationship between the output frequency and the applied voltage is known as the pushing characteristic; the relationship between the output frequency and external loading of the slow wave structure and, hence, the rf excitation is known as the pulling characteristic. The magnetron is a one port device and its operation can be influenced by having injected power on this port which is ordinarily for output.

Recently the authors [19,20] have demonstrated the phase locking of a $2.45 \mathrm{GHz}$ "cooker" magnetron with injection power $40 \mathrm{~dB}$ below the output power and without rigorous restrictions on power supply voltage ripple, load fluctuations, and thermal stabilization of the anode or restriction of the cathode heater power. This was achieved by injection locking the magnetron while its frequency was stabilized with a phase locked loop acting on the anode current and utilizing the magnetron frequency pushing characteristic. Note that the output frequency of most electrically excited oscillators depends on the supply current and voltage. When a magnetron is injection locked, there is a phase difference between the injection signal and the output that depends on the frequency difference between the injection signal and the natural frequency for the magnetron [9]. Nominally the phase difference $\phi$ is given as

$$
\sin \phi=2 Q \sqrt{\frac{P_{\text {out }}}{P_{\text {inj }}}} \frac{\omega_{d}-\omega_{o}(t)}{\omega_{d}},
$$

where $P_{\text {inj }}$ is locking power, $P_{\text {out }}$ is output power, $Q$ is the loaded $Q$ factor of the magnetron, $\omega_{d}$ is the angular frequency of the injection signal, and $\omega_{o}$ is the instantaneous natural angular frequency of the magnetron. Variation of $\omega_{o}$ comes from magnetron dimensional changes with temperature, anode current fluctuations (with associated voltage fluctuations), and possibly variations in cathode temperature. For the experiment reported here, current and hence voltage fluctuation came in the form of ripple from first the $42 \mathrm{kHz}$ chopper in the switched mode power supply, second from rectified mains ripple $100 \mathrm{~Hz}$ (UK), $120 \mathrm{~Hz}$ (US) on the dc power for the switch mode power supply, and third from the unrectified mains on the ac heater supply. The $3 \mathrm{~dB}$ bandwidth of the free running magnetron power output spectrum was typically $\pm 45 \mathrm{kHz}$ for $10 \mathrm{~W}$ heater power. Equation (1) predicts a phase jitter of $\pm 14^{\circ}$ for a magnetron with a loaded $Q$ of 200 when it is locked with a $-30 \mathrm{~dB}$ injection signal. When a magnetron is used to drive a superconducting accelerator cavity the precise phase and amplitude of its output is not the controlled set point, but the output must be varied so as to fix the phase and amplitude of the field in the cavity. As beamloading and microphonics alter the required drive amplitude and phase, then the important issue is how the injection locked magnetron behaves in a control loop.

\section{APPLICATION CONCEPT}

A key issue that needs to be addressed with respect to driving accelerator cavities with magnetrons is a method to control amplitude and phase simultaneously and independently. The output of a magnetron when operated as a reflection amplifier [21] has negligible dependence on the amplitude of the injection signal. Output phase and amplitude of the magnetron is determined by the anode current, the dc magnetic field, and the reflected power. The practicality of using these inputs for amplitude control is best considered within the context of an application. As a hypothetical but topical case, we consider the $40 \mathrm{~mA}$ high intensity beam option for SPL [1]. In this instance, a five cell cavity needs $880 \mathrm{~kW}$ of rf power at $704 \mathrm{MHz}$ for $1.2 \mathrm{~ms}$ and with a repetition rate of $50 \mathrm{~Hz}$. In principle, the 
anode current can be actively varied in response to a control signal during a pulse at the power levels concerned. This introduces considerable complexity into the high voltage modulator. Some degree of current variation during a pulse is more straightforward if the variation has been planned during the modulator charging cycle. Control of the magnetic field in the magnetron gets difficult on a submillisecond time scale as this will involve counteracting a large reactive electromotive force to ramp a current. Control of the reflected power also gets difficult on a submillisecond time scale as a relatively large mechanical stub has to be moved to a new position with a high accuracy.

The scheme that would probably be adopted for rapid and actively controlled variation of amplitude and phase during the pulse is to combine outputs of two magnetrons with opposite phase shifts each side of the desired output phase (the outputs should be considered as contributions after the combiner which will add $90^{\circ}$ on one path) such that the combined phase is equal to the desired output as illustrated in Fig. 1. By altering the two magnetron phase shifts, it is then possible to rapidly alter the resultant amplitude. The phase offset of these two magnetrons needs to be just sufficient so that when the two magnetrons are brought together they will give sufficient additional power for compensation of the variations in beamloading arising from unexpected bunch charge variations and microphonic detuning. In this scheme we note that the magnetrons are isolated by circulators and a magic tee. This isolation needs to be such that output from one magnetron reaching the other is somewhat smaller than the injection signal. One would design for an isolation better than $50 \mathrm{~dB}$. With adequate isolation any tendency for one magnetron to pull the other magnetron will be swamped by the injection signal.

Combining power from two magnetrons in the manner described clearly reduces efficiency. This loss in efficiency can be kept small if the short time scale amplitude fluctuations are small. The magnetron output can be varied by large factors on longer time scales while keeping the efficiency high by varying the anode current and adjusting

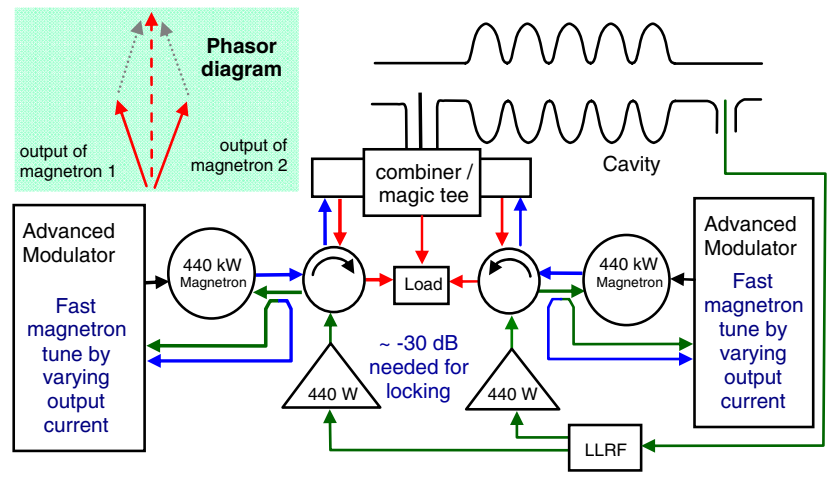

FIG. 1. Amplitude control scenario utilizing two magnetrons operating with a variable phase offset.

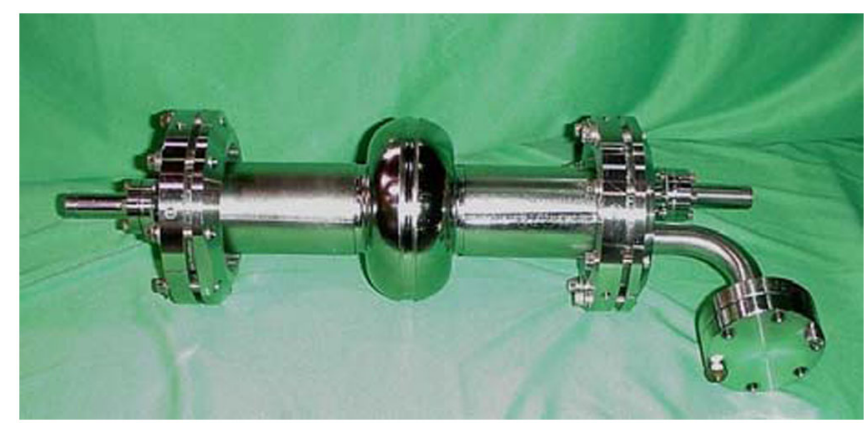

FIG. 2. Single cell $2.45 \mathrm{GHz}$ niobium cavity.

either the transverse magnetic field or cavity tuning rods to maintain the correct natural frequency for the magnetron.

The $440 \mathrm{~kW}$ magnetrons at $704 \mathrm{MHz}$ with a $10 \%$ duty cycle shown in this proposed system present no special developmental issues.

The first step to realizing a complete solution is the demonstration that a single superconducting cavity with a representative external $Q$ factor can be driven with accurate phase control from a magnetron. Rapid amplitude control is not addressed directly by the experiments undertaken here. In this paper we describe test results first for a superconducting cavity and then for a copper cavity.

\section{SUPERCONDUCTING CAVITY}

The superconducting test was conducted with a single cell niobium cavity whose acceleration mode was made at $2.45 \mathrm{GHz}$ and whose intrinsic quality factor $Q_{o}(2 K)=$ $5.22 \times 10^{9}$. Figure 2 is a photograph of the cavity. Prior to manufacturing the niobium cavity an identical cavity was manufactured from copper. Phase control experiments were also conducted with the copper cavity.

The cavity had a simple coaxial input coupler at the right end and a similar output coupler at the left end. The tests were conducted in a vertical cryostat at $2 \mathrm{~K}$. The input coupler was adjusted to give a fixed external $Q$ of $2.6 \times 10^{6}$ as would be typical for an accelerator with some beam loading. The output coupler was set to have an external $Q$ of $2.2 \times 10^{10}$. Controlled levels of microphonics could be introduced to the top of the Dewar.

\section{RF SYSTEM}

A key aspect of the experiment was to show that the phase of a superconducting cavity could be accurately controlled by manipulation of the phase of the locking signal to a magnetron. The configuration of the high power rf circuit utilized and one configuration of the control circuitry is shown in Fig. 3. A more complex configuration of the control circuitry, where a control signal to the switched mode power supply was actively varied to minimize locking power, was also investigated and is shown later in Fig. 12. 


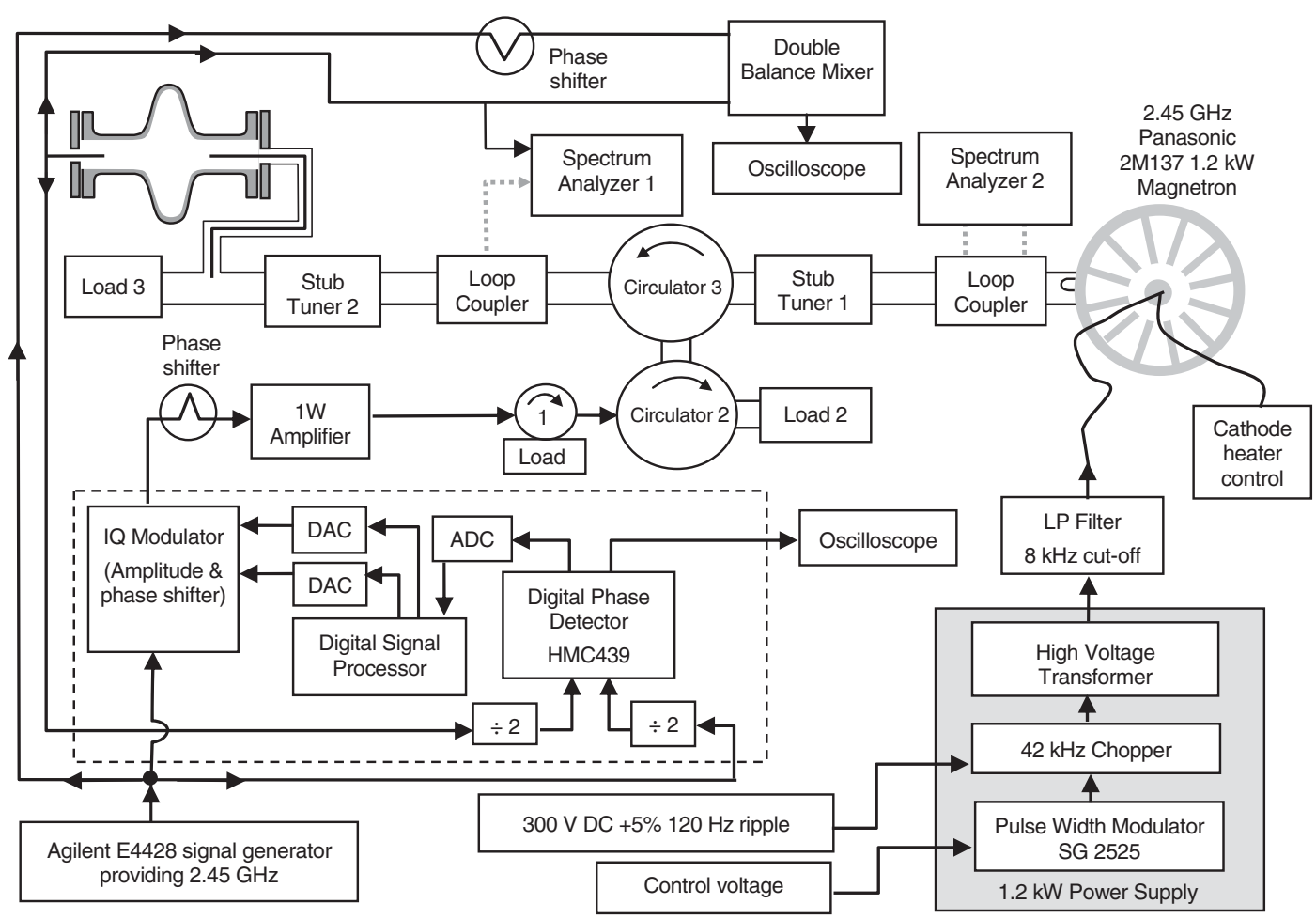

FIG. 3. Low and high level rf control layout of the magnetron phase lock to a superconducting rf cavity.

The magnetron used for the tests was a Panasonic $2 \mathrm{M} 137,1.2 \mathrm{~kW}, 12$ vane cooker type. Experimental results presented here were taken with magnetron output powers near to $530 \mathrm{~W}$. This level of power could potentially cause damaging arcs in the rf cable going to the Dewar or excessive heating for $\mathrm{cw}$ operation in a noncooled copper cavity. Consequently, the coupling arrangement between the cavity and the waveguide was set up to reduce the amount of power that could be delivered for respective normal conducting and superconducting tests.

The layout for operation with the superconducting cavity had a $10 \mathrm{~m}$ coaxial cable between the waveguide and the cavity input coupler to allow operation in a vertical cryostat. For operation with the copper cavity a short direct coaxial coupling was used. The coupling between the coaxial line and the copper cavity was optimized for maximum power transfer; however, the coupling between the coaxial line and the waveguide was adjusted for operation with forward powers not exceeding $60 \mathrm{~W}$.

The coupling between the coaxial line and the superconducting cavity was set to be considerably less than its coupling to the waveguide. For operation with this cavity, we note that manipulation of stub tuner 2 only gave a small variation in the cavity's external $Q$ factor as previously quoted. Forward power to the superconducting cavity was only $2.4 \mathrm{~W}$. The corresponding gradient in the superconducting cavity was $0.93 \mathrm{MV} / \mathrm{m}$. We note that the purpose of the tests were not to demonstrate high gradient.

For both cavity instances operation without stub tuner 2 lets all power leaving the cavity travel by low reflection routes to load 2 and load 3 so that external $Q$ is determined primarily by the coupling at the cavity rather than coupling at the waveguide.

For both configurations the dc cathode voltage for the magnetron was supplied from a commercial switched mode power supply modified so that the pulse width modulator could be controlled externally. For industrial applications the pulse width modulator is operated in a constant current control loop. With an external control, one has many options including a fixed modulation or operation at constant frequency utilizing the magnetron's pushing characteristic. Constant natural frequency control of a magnetron without a cavity (superconducting or normal) has been described previously $[19,20]$.

For the tests described here with a superconducting cavity, the best cavity phase control was obtained using the configuration of Fig. 3 where the reference oscillator was an Agilent E4428 source and the switched mode power supply received a steady pulse width modulation. The magnetron is operated as a reflection amplifier [21]. The source signal from the Agilent E4428 is split three ways. One signal goes as a reference to a double balanced mixer on the cavity output coupler to make high resolution phase measurements. The second signal is taken as a reference for the phase control loop. The third signal is used to provide the magnetron injection signal after its phase has been adjusted by the in-phase/quadrature (IQ) modulator. The IQ modulator is part of the cavity phase control loop. The injection power is amplified to $1 \mathrm{~W}$ and then injected into the magnetron via three circulators. Circulator 3 separates 
the magnetron output from the injected input. Circulator 2 diverts reflected power from the cavity away from the injection source to load 2. As the high power circulators have an isolation as little as $-20 \mathrm{~dB}$, an additional low power circulator 1 is used to protect the $1 \mathrm{~W}$ amplifier.

The magnetron's injection input and power output are through the same waveguide port. Adjacent to the magnetron's output port is a $-60 \mathrm{~dB}$ directional coupler and then a three stub tuner 1 . This tuner is used to alter the loading of the magnetron, i.e., its effective external $Q$ factor.

In the absence of an injection signal, adjustment of the magnetron anode current changes both the output power and the output frequency by frequency pushing. Adjustment of the magnetron loading (reflected power) also alters power output and frequency by frequency pulling but in a different ratio. By simultaneous adjustment of the anode current and the reflected power, it is possible to manipulate the frequency through a $1 \%$ range and the forward power from about $25 \%$ to $100 \%$ of full magnetron power.

If an injection signal is introduced when the natural frequency of the magnetron is close to that of the injection signal, then the magnetron's output frequency will become locked to the injection signal with a phase shift. During injection locked operation, the magnetron's natural frequency must be kept close to the frequency of the injection signal so as to minimize locking power as determined by the Adler equation (1) [11].

The control system for cavity phase illustrated with a dashed box in Fig. 3 is a generic system appropriate for a wide range of frequencies [22]. The phase was measured using a Hittite HMC439QS16G digital phase detector in conjunction with HMC437MS dividers. The benefit of this approach is its excellent phase linearity and, hence, any offset can be varied accurately during operation. The output from the Hittite digital phase detector is an analogue voltage which was sampled by an analogue to digital converter (ADC). The sampling speed was about 1 mega samples per second. The sampling speed was constrained by the rate at which the digital signal processor could implement the control algorithm for cavity phase.

The measurement circuit for the amplitude of the cavity probe signal is not shown in Fig. 3 as the cavity amplitude was not managed by the controller in the digital signal processor for this experiment. The injection signal for the magnetron is controlled by IQ modulation. The phase shift imparted by the IQ modulator determines the phase of the power delivered to the cavity. The controller modifies the phase of the injection signal to the magnetron in response to the phase of the field measured by the cavity probe. Proportional integral control was applied to cavity phase and the required output was converted to IQ components for the IQ modulator by the digital signal processor.

The phase of the cavity field is disturbed by microphonics and the noise arising from the magnetron source.
As has been said, the frequency and, hence, the phase of the output of a magnetron is sensitive to cathode voltage, heater voltage, and reflected power. Reflected power stays almost fixed when the magnetron is protected with a circulator. A cooker-type magnetron has a coiled filament cathode that is heated by an ac current floated at the cathode potential. Driving this heater current through the resistance of the filament adds a few volts drop at $60 \mathrm{~Hz}$ along the cathode whose dc offset is about $-4000 \mathrm{~V}$ with respect to the anode. Additionally, the cathode voltage has ripple at the switched mode power supply chopping frequency of $42 \mathrm{kHz}$ and has $120 \mathrm{~Hz}$ ripples from the dc supply. In Fig. 3 the low pass filter after the switched mode power supply reduces $42 \mathrm{kHz}$ ripple from the chopper.

\section{SUPERCONDUCTING OPERATION}

For the configuration shown in Fig. 3 where there is no automatic control on the natural frequency of the magnetron, the operating procedure was as follows. With the magnetron power supply off and the digital signal processor inactive an injection signal was introduced. The injection signal fills the magnetron and is established at the input coupler of the superconducting cavity. As the cavity does not have a tuner, the frequency of the injection signal is adjusted until the cavity is filled and produces a measurable output as shown in Fig. 4.

A $-61 \mathrm{~dB}$ microphonic $(-37 \mathrm{~dB}$ below the center frequency) at $\pm 31 \mathrm{~Hz}$ can be observed. The center frequency is noted. The injection signal is then switched off and the magnetron energized at full heater power. After start up the heater power is reduced to a level of about $15 \%$ to $30 \%$ of that required for start up. In this range the cooker-type magnetron exhibits a relatively low noise output [23]. The power output and frequency of the magnetron is then adjusted by varying its loading (reflected power)

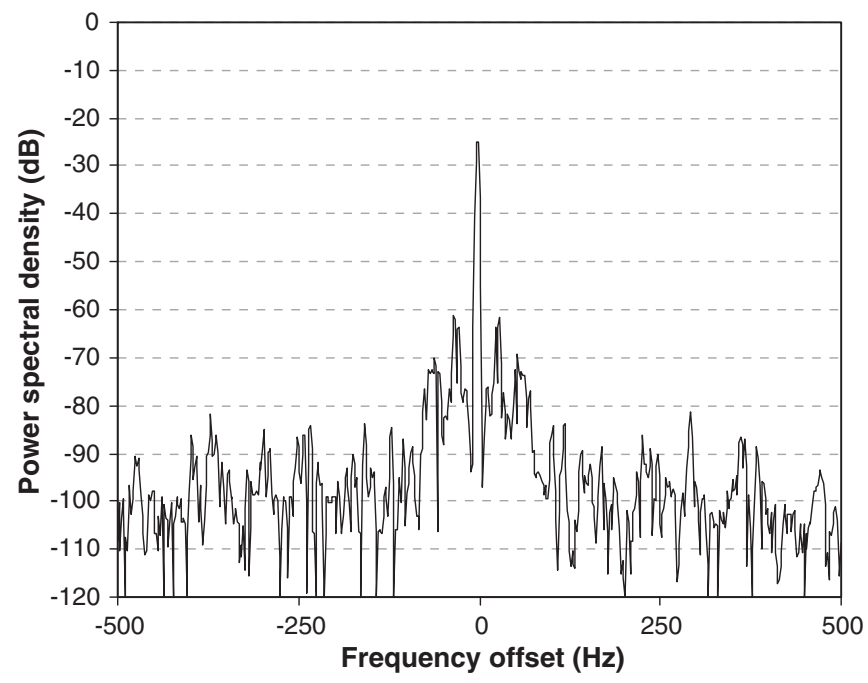

FIG. 4. Cavity spectral output (dB) with only injection signal as input. Resolution bandwidth $\mathrm{RBW}=3 \mathrm{~Hz}$. 
and the control voltage on the switch mode power supply as previously described. Typically, the anode current was adjusted and maintained near to $250 \mathrm{~mA}$ so that the output power was $540 \mathrm{~W}$ plus or minus $20 \mathrm{~W}$. Reflected power and anode current were adjusted together until the magnetron frequency was less than $10 \mathrm{kHz}$ from the center frequency of the superconducting cavity as measured just previously. Final adjustment was made once the magnetron's temperature had stabilized. One notes that this complex procedure is not necessary when automatic control is established between the magnetron frequency and the anode current. In this instance, one only adjusts the stub tuner 2 to get the desired power output.

Once the magnetron has stabilized within $\pm 1 \mathrm{MHz}$ of cavity resonance frequency the injection signal is turned on. At this instant the magnetron locks and behaves as a constant output amplifier, i.e., the phase of the output follows the phase of the input. Previous accurate adjustment of the injection signal frequency enables the filling of the narrow bandwidth resonance superconducting cavity. The center frequency of the cavity is very stable under the $2 \mathrm{~K}$ helium pressure in $\pm 2 \mathrm{~Hz}$.

\section{EXPERIMENTAL RESULTS FOR SUPERCONDUCTING CAVITY}

Figure 5 shows the spectral output once the magnetron is powered and injection locked but without the digital signal processor (DSP) controlling the cavity phase.

The resonance peak has increased from $-24.8 \mathrm{~dB}$ as seen in Fig. 4 to $-3.8 \mathrm{~dB}$ as seen in Fig. 3 which is an increase of $21 \mathrm{~dB}$. (The result of Fig. 4 did not have the amplification of the injection signal added by the magnetron.) The phase jitter at the electricity main supply (mains) power frequency of $60 \mathrm{~Hz}$ and its harmonics (120, 180, $240 \mathrm{~Hz}$, etc.) emanating from the magnetron power supply and the cathode heater supply have now been introduced to the cavity.

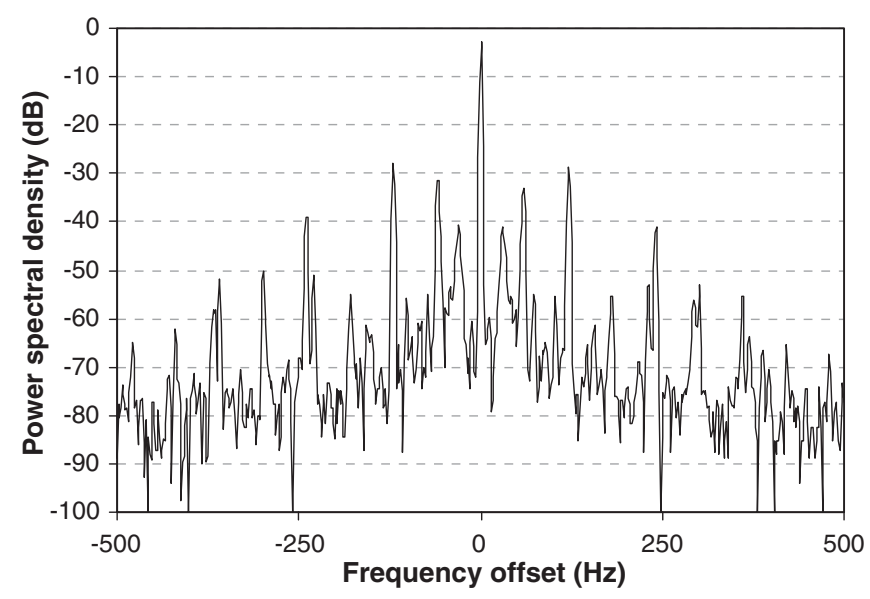

FIG. 5. Cavity spectral output (dB) with injection locked magnetron and without DSP control, RBW $=3 \mathrm{~Hz}$.

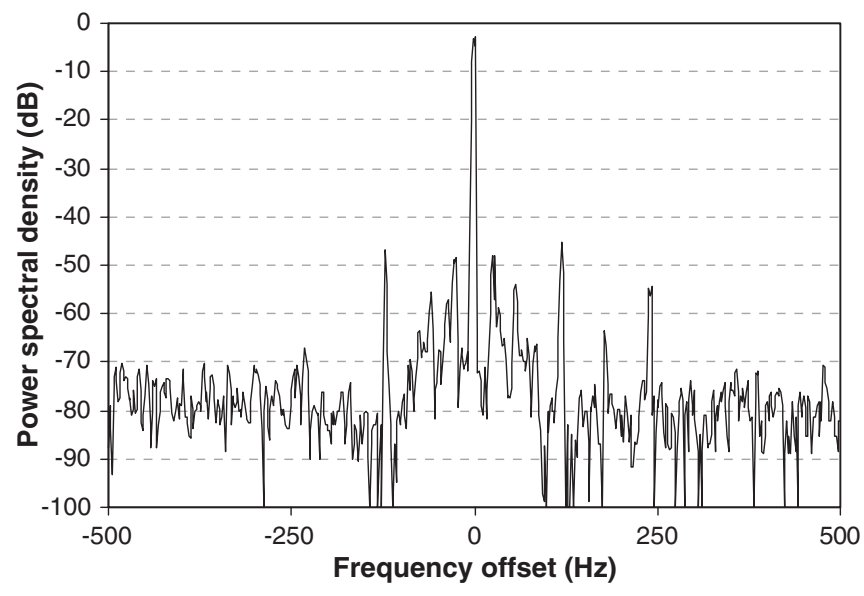

FIG. 6. Cavity spectral output (dB) with injection locked magnetron and DSP control, RBW $=3 \mathrm{~Hz}$.

The spectrum after application of the digital signal processor control as shown in Fig. 6 reduces the microphonic phase jitter at $31 \mathrm{~Hz}$ from $-42.3 \mathrm{~dB}$ to $-48.7 \mathrm{~dB}$ $(6.4 \mathrm{~dB})$, it reduces the $60 \mathrm{~Hz}$ ripple from $-34.8 \mathrm{~dB}$ to $-55 \mathrm{~dB}(20.2 \mathrm{~dB})$, and the $120 \mathrm{~Hz}$ ripple from $-28.5 \mathrm{~dB}$ to $-46.2 \mathrm{~dB}(17.7 \mathrm{~dB})$. Attempts to improve noise reduction by increasing the control loop gain resulted in loss of lock.

For the magnetron to correct a phase error it has to run at a frequency offset for a small period of time. Running at a frequency offset might move the magnetron to a frequency where more injection power is needed to maintain the lock. Increasing the injection power from 1 to $2 \mathrm{~W}$ did not enable any noticeable increase in gain or indeed any further reduction in noise. This indicated that other aspects of the control system were the limiting factor.

As the controller was implemented digitally with an associated time delay, there is a maximum gain beyond which a feedback controller becomes unstable. For simple proportional control this stability limit occurs when the gain equals the reciprocal of the product of the time delay and the bandwidth. A more complicated formula exists for PI control [24]. The effective bandwidth is that of the superconducting cavity as this is much smaller than that of the magnetron and other system components. It is likely that the loss in lock at high gain was associated with control instability arising from the processing time delay. Alternatively, one might also consider the time delay associated with the slew rate of the injection locked magnetron. The DSP processing time delay was approximately $1 \mu \mathrm{s}$ while Fig. 14 shows the magnetron reacting to phase changes on a scale shorter than $1 \mu \mathrm{s}$.

Figure 7 shows a time domain record of control performance. The phase to voltage conversion from the double balance mixer had been calibrated to be $10^{\circ} / \mathrm{V}$. Comparing traces in Fig. 7 the peak-to-peak phase noise of the cavity output is reduced from $50^{\circ}$ peak to peak to $2^{\circ}$ peak to peak $\left(0.95^{\circ} \mathrm{rms}\right)$. 


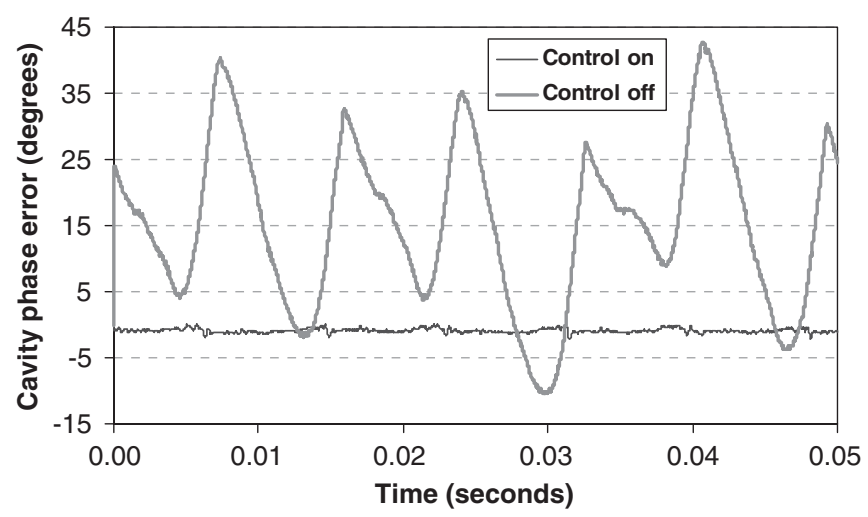

FIG. 7. Phase jitter measurement from the cavity output.

\section{Results with added microphonics}

The control action was investigated further by deliberately introducing additional microphonics by means of a shaker placed on the lid of the cryostat operated at $50 \mathrm{~Hz}$. Figure 8 shows the output spectrum from the cavity with the shaker applied when powered by the injection locked magnetron.

Comparing Fig. 8 with Fig. 5, one notices that the central excitation of the cavity has reduced from $-3.8 \mathrm{~dB}$ to $-6.3 \mathrm{~dB}$; this was for the same cavity tuner position and the same magnetron output. This indicates that the shaker cycles the natural frequency of the cavity by an amount which is a significant proportion to the bandwidth. The spectrum shows the $50 \mathrm{~Hz}$ peak from the shaker adjacent to the $60 \mathrm{~Hz}$ peak from the power supply. One notes that the relative heights of the $60 \mathrm{~Hz}$ peak and its harmonics are marginally increased with respect to Fig. 5 while the microphonics peak at $30 \mathrm{~Hz}$ is marginally reduced by $1 \mathrm{~dB}$.

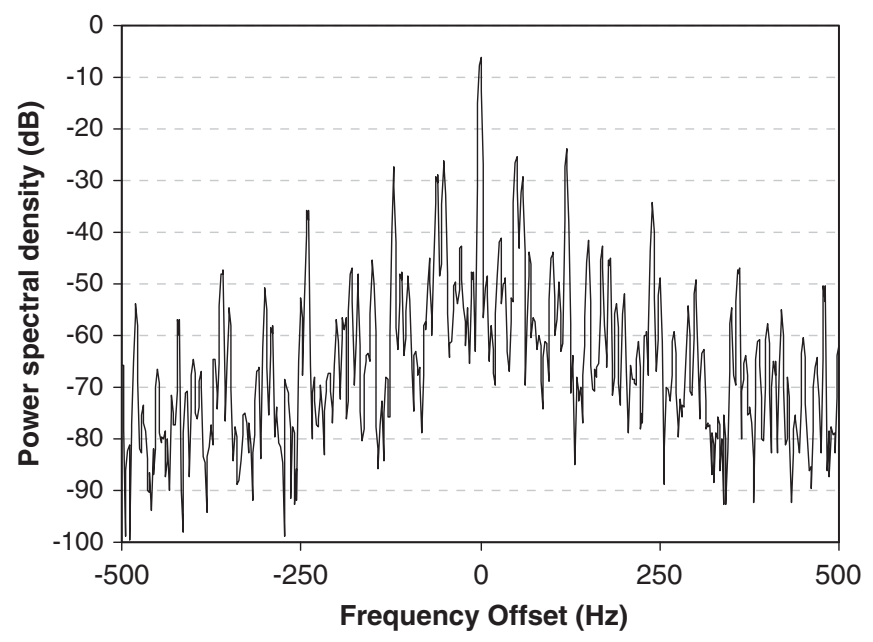

FIG. 8. Spectral output (dB) of cavity with shaker for injection locked magnetron and without DSP control, RBW $=3 \mathrm{~Hz}$.

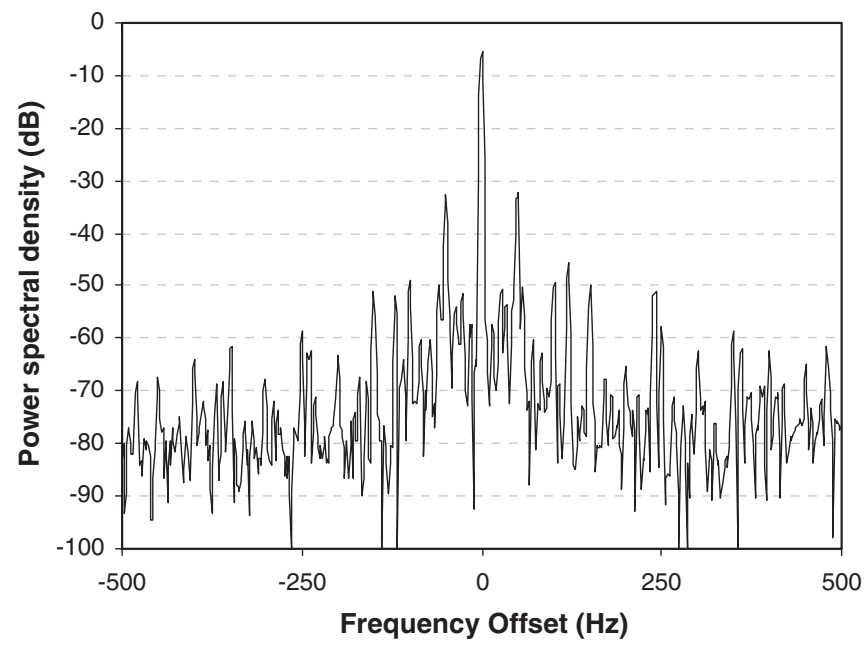

FIG. 9. Cavity spectral output $(\mathrm{dB})$ with shaker for injection locked magnetron with DSP control, RBW $=3 \mathrm{~Hz}$.

Figure 9 shows the output spectrum once the active phase control is applied. The center peak rises from $-6.3 \mathrm{~dB}$ to $-5.3 \mathrm{~dB}$. The $50 \mathrm{~Hz}$ peak caused by the shaker is reduced from $-25.3 \mathrm{~dB}$ to $-33.3 \mathrm{~dB}(8 \mathrm{~dB})$, the $60 \mathrm{~Hz}$ peak from the power supply is reduced from $-29.3 \mathrm{~dB}$ to $-50 \mathrm{~dB}(20.7 \mathrm{~dB})$, and the $120 \mathrm{~Hz}$ peak is reduced from $-27.2 \mathrm{~dB}$ to $-51.8 \mathrm{~dB}(24.6 \mathrm{~dB})$.

\section{SOURCE NOISE AND MEASUREMENT}

In Fig. 3 it was seen that the double balanced mixer phase measurements were made by comparing a signal which was taken from the source with a signal that had been delayed by the transmission through the IQ modulator, the $1 \mathrm{~W}$ amplifier, the magnetron, and finally the superconducting cavity. By far the largest delay is that through the superconducting cavity whose loaded $Q$ was about $2.2 \times 10^{6}$. The group delay through the cavity depends on the frequency. Approximating the cavity as the parallel LCR circuit for a single resonance then a little analysis gives the phase shift and, hence, the group delay is derived as

$$
\tau(\omega)=-\frac{\partial \varphi}{\partial \omega}=-\frac{\partial}{\partial \omega} \tan ^{-1}\left[Q_{L}\left(\frac{\omega_{c}}{\omega}-\frac{\omega}{\omega_{c}}\right)\right],
$$

where $\omega_{c}$ is the cavity center angular frequency. Hence,

$$
\tau(\omega)=\frac{\frac{Q_{L}}{\omega_{c}}\left(\frac{\omega_{c}^{2}}{\omega^{2}}+1\right)}{1+Q_{L}^{2}\left(\frac{\omega_{c}}{\omega}-\frac{\omega}{\omega_{c}}\right)^{2}} .
$$

The $-3 \mathrm{~dB}$ bandwidth of the cavity is given as $\frac{\omega_{c}}{2 \pi Q_{L}}$.

Spectral components of the noise which are further than half a bandwidth from the center frequency have a little 


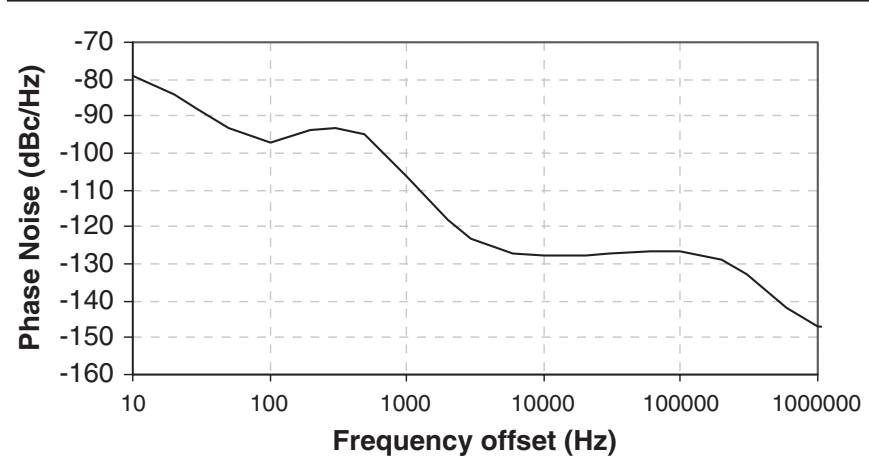

FIG. 10. Replot of Agilent E4428 signal phase noise figure at a carrier frequency of $2.2 \mathrm{GHz}$.

delay but considerable attenuation. Spectral components of the noise which are closer to the center frequency than half the bandwidth have a lot of delay but little attenuation. The double balanced mixer output is proportional to the amplitude of each Fourier component of the rf input. While the source is a direct input to the reference input of the double balanced mixer, noise outside the bandwidth of the superconducting cavity is strongly attenuated in the mixer's output. The overall effect is that the time domain cavity phase jitter measurement data has added source noise, as would be attenuated by the cavity even though the reference signal is not filtered by the cavity. The underlining jitter with source noise subtracted is limited by the extent to which the controller can correct for microphonic and magnetron disturbances.

Figure 10 replots the phase noise data for the Agilent E4428 at $2.2 \mathrm{GHz}$ from the manufacturer's datasheet [25]. From this data we compute that the jitter contribution from the source for frequencies greater than $20 \mathrm{~Hz}$ (which corresponds to the time domain plot of Fig. 7) was approximately 0.14 degrees rms ( 0.40 degrees peak to peak). As the controller is also using the source as its reference and has its own delay then, even if the controller were optimal, additional jitter would be introduced.

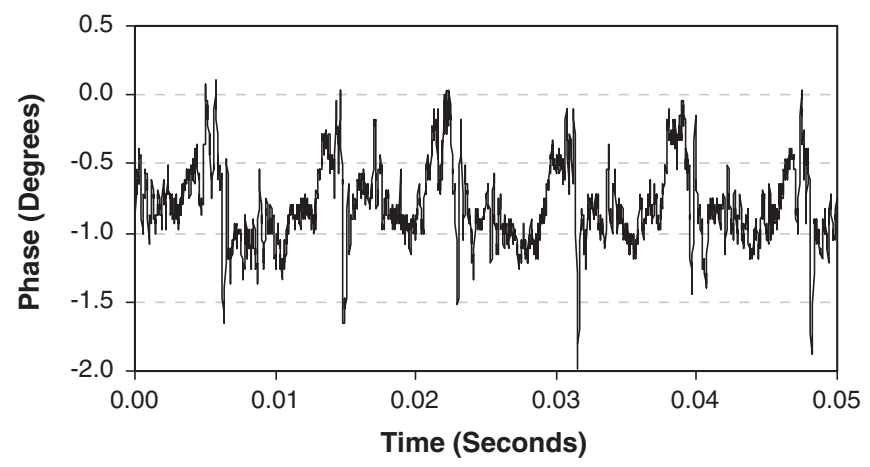

FIG. 11. Phase jitter measurement for the case with the DSP controller activated as shown in Fig. 7 but with an expanded vertical scale.
The ultimate performance we might have hoped to have measured, had we been able to increase controller gain without loss of lock, would have been at least 0.14 degrees rms against the 0.95 degrees that was achieved. Figure 11 replots the phase jitter observed with the cavity phase controller active on an expanded vertical scale.

The residual phase error has sharp peaks at $120 \mathrm{~Hz}$. These can be associated with the abrupt changes of phase of the uncontrolled cavity output in Fig. 7. From the results measured with the copper cavity shown later in Fig. 14, we see that the abrupt changes of phase are associated with corners of the sawtooth anode current. With more development effort on the power supply and the controller then better performance is certain to be possible.

It should be noted that the noise floor of the spectrum analyzer used for the spectral plots was only $-75 \mathrm{dBc}$ for the $1 \mathrm{kHz}$ range and, hence, it could not be used to accurately determine the phase jitter to values better than about 1.0 degrees rms in the time domain.

\section{CLOSED LOOP FREQUENCY CONTROL}

Figure 12 shows the preferred alternative control configuration where the frequency of the natural magnetron is stabilized. This configuration was used with the normal conducting copper cavity but was unsuccessful with the superconducting cavity because the voltage controlled oscillators (VCOs) on board were picking up $60 \mathrm{~Hz}$ interference from the local environment to a level that pushed the phase shift of the superconducting cavity just outside the locking range. As the vertical cryostat facility was a permanent installation with restricted access, the problem could not be resolved during the scheduled period for the vertical test.

\section{RESULTS FOR COPPER CAVITY}

Figure 13 shows injection locking results for the copper cavity with rf control implemented using active phase control. The top trace is anode current ripple $(\sim 13 \%)$, the middle trace is the phase error without active phase control ( $45^{\circ}$ peak to peak), and the lower trace is the phase error with active phase control $\left(7.5^{\circ}\right.$ peak to peak). The resulting phase error comes partly from the $42 \mathrm{kHz}$ switch mode power supply frequency that is transmitted by a normal conducting cavity. The $100 \mathrm{~Hz}$ component is coming from rectified UK mains (this trace was recorded in the UK).

A better phase control result was achieved with the superconducting cavity in Fig. 7 than with the normal conducting cavity. Filtering by the superconducting cavity of the $42 \mathrm{kHz}$ allowed a higher effective gain to be used in the control loop.

The magnetron bandwidth needs to be considered when optimizing cavity control. Figure 14 shows response of the magnetron to a shift in the drive phase. The upper trace 


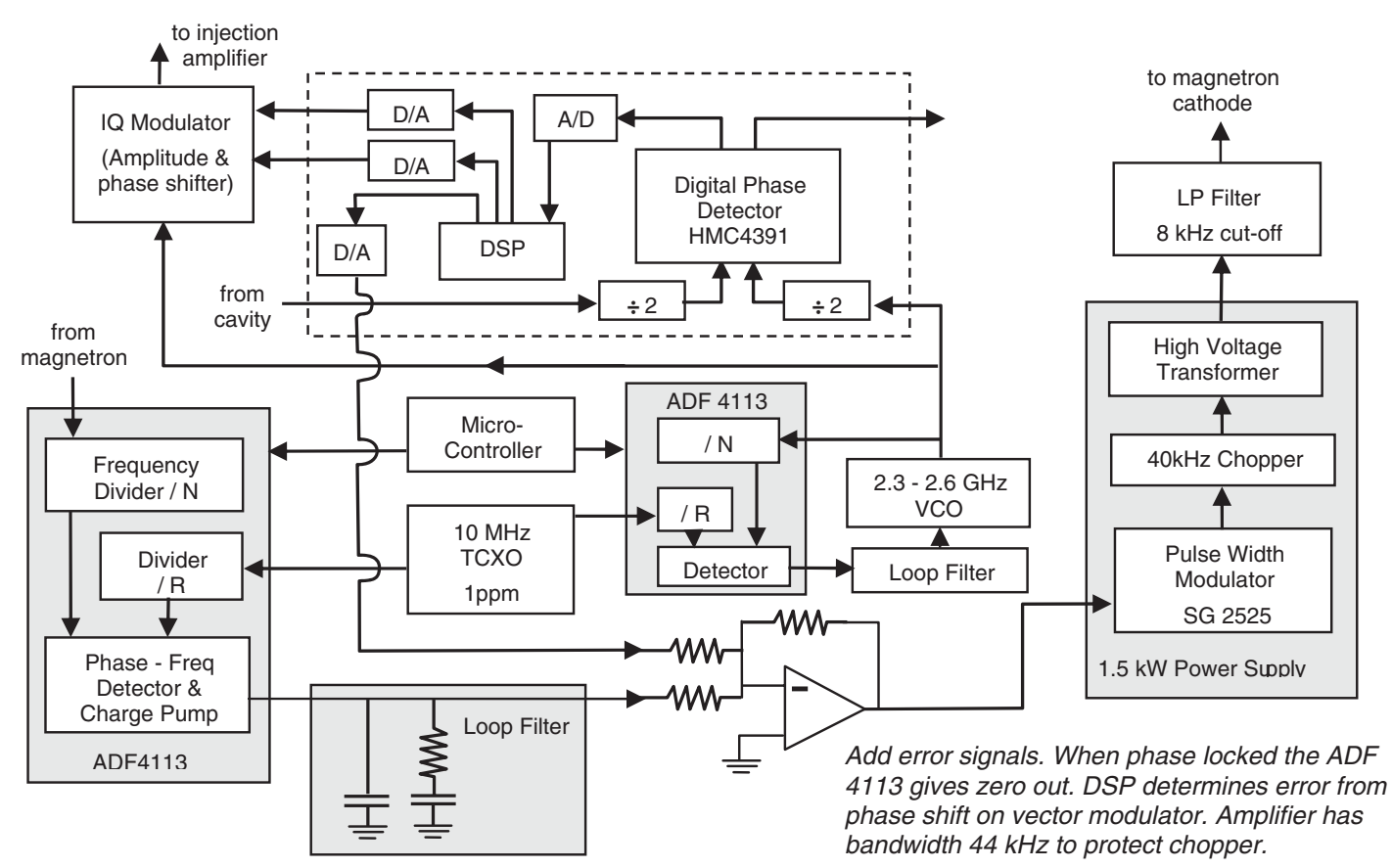

FIG. 12. Active control arrangement with magnetron in a phase lock loop.

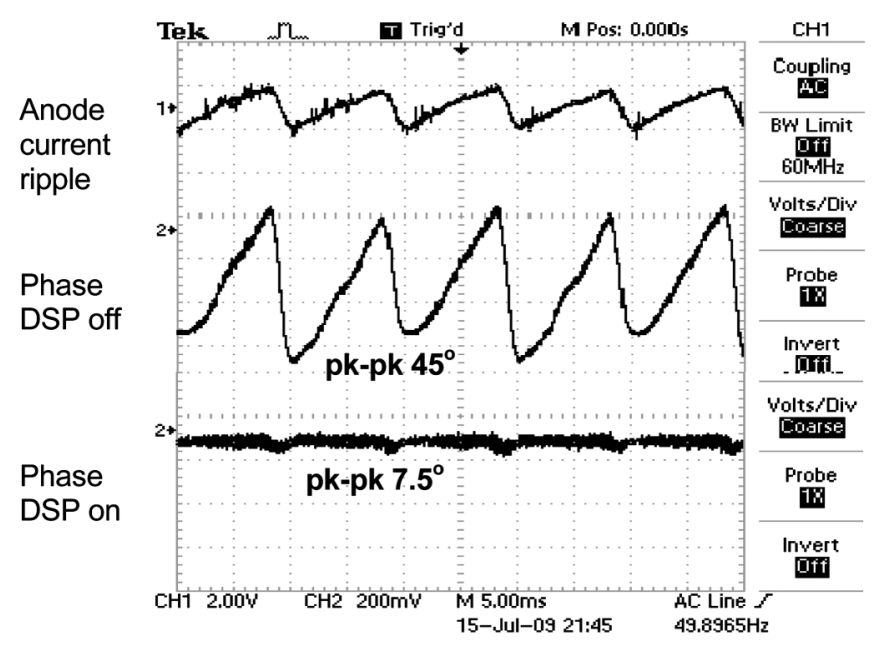

FIG. 13. Middle and lower traces show phase jitter with and without fast feedback, respectively. The top trace is current ripple.

shows the drive phase being switched between $0^{\circ}$ and $90^{\circ}$ at a frequency of $2 \mathrm{MHz}$. The lower figure is the magnetron's phase response measured by the double balanced mixer.

The precise form of the response depends on the load impedance, the injection power, and the cathode heater power. The major divisions on the horizontal scale represent $500 \mathrm{~ns}$; hence, the response time is of the order of $200 \mathrm{~ns}$ for a $90^{\circ}$ phase shift. This bandwidth would restrict the phase control performance for short pulse normal conducting accelerator applications but

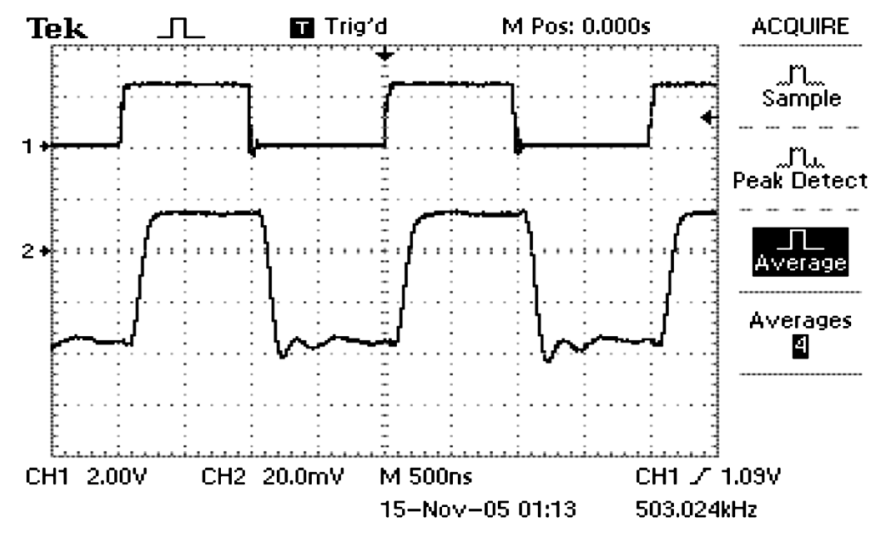

FIG. 14. Magnetron response to a $90^{\circ}$ phase shift in its locking signal.

is more than adequate for superconducting accelerator applications.

\section{CONCLUSIONS}

This paper has suggested a method for employing magnetrons as sources of long pulse or continuous wave $\mathrm{rf}$ power in order to individually drive accelerator cavities (typically multicell) along a beam line where accurate relative phase synchronization and accurate amplitude control is required. Where accelerator cavities in a linac need individual phase and amplitude control, the application of magnetrons is likely to give large capital cost and efficiency savings. Along a superconducting linac we note that microphonics and thermal drift cause differential 
phase errors between cavities and viable beamloading causes amplitude errors in cavities. These errors must be compensated by a control loop of which the high power rf amplifier forms a part.

Magnetron reflection amplifiers operate at saturation and, hence, output amplitude control is only possible by varying the anode current or the transverse magnetic field or the load impedance. As these control methods may be too slow to react to cavity amplitude fluctuations arising from variable beamloading, a solution for fast amplitude control by combining the outputs from two magnetrons each with a variable phase shift has been proposed. In combination, the average phase contribution from the two magnetrons (after the combiner) determines the rf phase of the power delivered to the accelerator cavity and the effective phase difference of the two magnetrons (after the combiner) determines the reduction in amplitude of the rf power. Phase and amplitude variation of the $\mathrm{rf}$ power to accelerator cavities can be made independently.

A key challenge to driving superconducting accelerator cavities with magnetrons is gaining the ability to rapidly manipulate the phase of a magnetron's rf output. This manipulation must be on a sufficiently small time scale to allow compensation of (open loop) cavity phase shifts arising from microphonics. This manipulation must also be on a time scale that allows compensation of (open loop) amplitude fluctuation arising from beamloading using the two magnetron scheme. The experimental work presented here has demonstrated that a magnetron will operate successfully in a control loop that maintains the phase of a superconducting cavity.

We note that the results obtained were limited by the performance of the commercial power supply that was modified for the experiment. One anticipates that results can be improved by developing a bespoke power supply with reduced dc ripple.

Results have also been presented for a normal conducting cavity. The special configuration of the control circuits allowed the magnetron to be operated in an injection locked state in the control loop during the period where cavity's temperature was still stabilizing. Unfortunately, the VCO utilized for this control circuitry was not sufficiently stable to stay within the bandwidth of the superconducting cavity and, hence, the best results for the superconducting cavity were obtained with an Agilent source once the operation of the magnetron had stabilized and with the magnetron anode current control disabled.

The key to getting a magnetron to run as amplifier with a relatively low noise factor is achieving stability of the operating conditions, stability of the power supply, and having the ability to adjust the heater current after preheat.

The work here leaves us far from demonstrating the viability of using a magnetron in a large accelerator facility. Proceeding to a real application, the initial problem one faces is that long pulse, high power magnetrons operating at accelerator frequencies do not exist. The next step is therefore to select an application and develop a magnetron. The magnetron development must focus high efficiency and high reliability. The external $Q$ factor of the magnetron anode structure will be relatively low so that the power required for injection locking is not excessive. One might also shape the pushing curve so that the anode current can be used to manipulate the natural frequency of the magnetron on a submillisecond time scale. Once the magnetron exists its characteristics can be accurately measured and a power supply can be developed. At the anticipated power levels of $500 \mathrm{~kW}$, the power supply costs are very high. Achieving a full system with two magnetrons coupled together providing amplitude and phase control on a superconducting cavity at the MW power level clearly requires major investment.

\section{ACKNOWLEDGMENTS}

This work has been supported by the DOE Contracts No. DE-AC05-06OR23177 and STFC No. ST/G008248/1.

[1] E. Benedetto, Report No. CERN-BE-2009-037.

[2] O. Brunner, Report No. CERN-AB-2008-067.

[3] G.H. Hoffstaetter, in Proceedings of the 9th European Particle Accelerator Conference, Lucerne, 2004 (EPSAG, Lucerne, 2004), pp. 497-499.

[4] L. Harwood, in Proceedings of the 20th Particle Accelerator Conference, Portland, OR, 2003 (IEEE, New York, 2003), pp. 586-588.

[5] S. Lenci and P. Eisen, in Proceedings of the 9th European Particle Accelerator Conference, Lucerne, 2004 (EPSAG, Lucerne, 2004), pp. 1114-1116.

[6] R. G. Carter, in Proceedings of the CERN Accelerator School "RF for Accelerators" (Ebeltoft, Denmark, 2010).

[7] R. G. Carter, in IEEE International Vacuum Electronics Conference, 2009 (IVEC '09) (IEEE, Piscataway, NJ, 2009), pp. 550-551, http://ieeexplore.ieee.org/stamp/ stamp.jsp?tp=\&arnumber $=5193458 \&$ isnumber $=5193336$.

[8] F. Gerigk et al., Report. No. CERN-2006-006.

[9] A. W. Hull, J. Am. Inst. Electr. Eng. 40, 715 (1921).

[10] F. R. Elder, Proc. IRE 13, 159 (1925).

[11] R. Adler, Proc. IRE 34, 351 (1946).

[12] T. Overett, E. Bowles, D. B. Remsen, R. E. Smith III, and G.E. Thomas, in Proceedings of the 1987 Particle Accelerator Conference (IEEE, Washington, DC, 1987), pp. 1464-1465.

[13] J. Benford, H. Sze, W. Woo, R. Smith, and B. Harteneck, Phys. Rev. Lett. 62, 969 (1989).

[14] T. A. Treado, T. A. Hansen, and D. J. Jenkins, in IEEE Particle Accelerator Conference (APS Beam Physics, San Francisco, California, 1991), pp. 702-704.

[15] S. C. Chen, G. Bekefi, and R. J. Temkin, in IEEE Particle Accelerator Conference (Ref. [14]), pp. 751-753.

[16] T. A. Treado, P. D. Brown, T. A. Hansen, and D. J. Aiguier, IEEE Trans. Plasma Sci. 22, 616 (1994). 
[17] Todd A. Treado, Paul D. Brown, and Aiguier Darrell, in Proceedings Intense Microwave Pulses, SPIE, 1993 (SPIE, Bellingham, WA, 1993), Vol. 1872, pp. 241-251.

[18] H. L. Thal and R.G. Lock, IEEE Trans. Microwave Theory Tech. 13, 836 (1965).

[19] I. Tahir, A. Dexter, and R. Carter, IEEE Trans. Electron Devices 52, 2096 (2005).

[20] I. Tahir, A.C. Dexter, and R. G. Carter, IEEE Trans. Electron Devices 53, 1721 (2006).
[21] J. Kline, IRE Trans. Electron Devices 8, 437 (1961).

[22] Dexter et al., EUROTeV-Report-2008-073, 2008.

[23] W.C. Brown, Proceedings of the 1st International Workshop on Cross-Field Devices (The University of Michigan, Ann Arbor, MI, 1995), p. 178.

[24] Dexter et al., EUROTeV-Report-2008-064, 2008.

[25] Agilent E4428C ESG Analog Signal Generator Data sheet, Characteristic SSB phase noise (measured), 2008, p. 10, http://www.agilent.com. 